Klaus Ridder

\title{
Theatrale Repräsentation religiösen und sozialen Wandels
}

\author{
Schauspiele in Nürnberg, Bern und Wittenberg - vor und nach \\ der Reformation
}

\section{Problemstellung}

Ein wichtiges Indiz für bedrohte Ordnungen sind hypothetische Beschreibungen des zu Erwartenden, wenn eine geltende Ordnung außer Kraft gesetzt oder eine als deviant gewertete Ordnung etabliert werden soll. Solche Entwürfe von Schadenserwartungen, im Tübinger SFB 923 >Bedrohte Ordnungen` wird dafür der Begriff >Bedrohungsszenarien` verwendet, sind ein wiederkehrendes Element der Kommunikation über Ordnungen im Zustand der Bedrohung. Der Aufsatz untersucht religiös akzentuierte Bedrohungsszenarien in vor- und nachreformatorischen Schauspielen; sie werden als Ausdrucksformen und Deutungen übergreifender sozial-ökonomischer und politisch-gesellschaftlicher Wandlungsprozesse verstanden. ${ }^{1}$ Um solche Zusammenhänge aufzuschließen, reicht es nicht aus, von der Gewissheit auszugehen, das Religiöse habe in Mittelalter und Früher Neuzeit die gesamte Gesellschaft und nahezu alle kulturellen Ausdrucksformen durchdrungen. Weiterführend erscheint eine integrale Analyse des Religiösen in seinem sozio-kulturellen Kontext, um eine schärfere

\footnotetext{
1 Die Argumentation greift die im Rahmen des Tübinger SFB 923 >Bedrohte Ordnungen ‘ aufgeworfenen Fragen nach der Qualifizierung von Bedrohten Ordnungen, nach ihrer Bewältigung und Reflexion sowie nach den Formen der Restituierung bzw. Neukonstituierung von Ordnung auf und diskutiert sie im Zusammenhang spätmittelalterlicher und frühneuzeitlicher Schauspieltexte. Zur Konzeption und zu den Analysekategorien des SFB vgl. Ewald Frie, Mischa Meier (Hg.): Aufruhr - Katastrophe - Konkurrenz - Zerfall. Bedrohte Ordnungen als Thema der Kulturwissenschaften. Tübingen 2014; Ewald Frie, Boris Nieswand: `Bedrohte Ordnungen als Thema der Kulturwissenschaften. Zwölf Thesen zur Begründung eines Forschungsbereichs. In: Journal of Modern European History 15,1 (2017), S. 5-15 und S. 31-35. Zur Bedrohungskommunikation vgl. Fabian Fechner, Tanja Granzow, Jacek Klimek, Roman Krawielicki, Beatrice von Lüpke, Rebekka Nöcker: We are gambling with our survival. Bedrohungskommunikation als Indikator für bedrohte Ordnungen. In: Ewald Frie, Mischa Meier (Hg.): Aufruhr Katastrophe - Konkurrenz - Zerfall (s. o.), S. 137-142.
}

Klaus Ridder, Tübingen 
Wahrnehmung der Interdependenzen und Verweiszusammenhänge zwischen Religion und Politik zu ermöglichen.

Exemplarisch werden drei Spiele hinzugezogen: das Nürnberger Fastnachtspiel Der Herzog von Burgund von Hans Folz (zwischen 1486 und 1494 entstanden), das Berner Fastnachtspiel Vom Papst und seiner Priesterschaft des Niklaus Manuel (1523 aufgeführt) sowie das in lateinischer Sprache 1538 in Wittenberg gedruckte Pammachius-Drama des Thomas Naogeorg. Durch diese Auswahl kommen zwei der wirkungsvollsten Bedrohungsszenarien - Jüdische Verschwörung und Konfessionelle Verketzerung - sowie die historische und mediale Situation vor und nach der Reformation in den Blick.

Inhaltlich geht es in den drei Stücken um Folgendes: Im Spiel des Hans Folz trifft der Herzog von Burgund während eines Besuchs der Stadt Nürnberg auf die Prophetin Sibilla, die ihm berichtet, einige Rabbiner behaupteten, ihr Messias sei gekommen und werde sie zur Weltherrschaft führen. Sibilla entlarvt den falschen Messias als Antichrist, als Betrüger. Daraufhin gesteht er alle geheimen Intrigen der Juden gegen die Christen. Dies führt zu deren Erniedrigung, Bestrafung und Ausweisung aus der Stadt.

Niklaus Manuels Fastnachtspiel beginnt mit der berühmten $>$ TotenfresserSzenes: Die Beerdigung eines reichen Bauern gibt Anlass, die Unsitte der Totenmessen und der Jahrzeiten (Seelenmessen), also die Ausbeutungspolitik der Gläubigen durch Klerus und Papst, offenzulegen. In nachfolgenden Szenen agiert der Papst als umjubelter Kriegsherr, der Feldzüge gegen christliche Länder vorbereitet. Sieben Bauern bringen schließlich ihren Protest gegen die papistischen Machenschaften zum Ausdruck und der Apostel Petrus legt dar, dass der Papst nicht sein Statthalter sei, sondern der Antichrist.

Auch Thomas Naogeorg arbeitet im Pammachius-Drama mit Antichrist-Motiven. Das Stück beginnt mit einer Szene im Himmel (Christus, Petrus und Paulus diagnostizieren die Weltlage) und spannt dann den Bogen von den Anfängen des Christentums als Staatsreligion (der Herrschaftszeit von Papst und Teufel) bis zu den Anfängen der Reformation (eine direkt auf Christus zurückgehende Maßnahme zur Rettung der Menschheit), nach der nur noch das Jüngste Gericht zu erwarten ist. $^{2}$

2 Hans Folz: »Der Herzog von Burgund « (F 88). Bearbeitet von Stefan Hannes Greil und Martin Przybilski. In: Nürnberger Fastnachtspiele des 15. Jahrhunderts von Hans Folz und aus seinem Umkreis. Edition und Kommentar. Hg. von Stefan Hannes Greil und Martin Przybilski, unter Mitarbeit von Theresia Biehl, Christoph Gerhardt und Mark Ritz. Mit einem Beitrag von Nikolaus Ruge. Berlin, Boston 2020, S. 171-201 (Text), S. 202-215 (Kommentar). Dazu zuletzt Beatrice von Lüpke: Nürnberger Fastnachtspiele und städtische Ordnung. Tübingen 2017, S. 103110; Niklaus Manuel: Werke und Briefe. Vollständige Neuedition. Hg. von Paul Zinsli, Thomas 
Die Schauspiele werden in kommunikations- und sozialgeschichtlicher Perspektive interpretiert. ${ }^{3} \mathrm{Zu}$ berücksichtigen ist, dass sowohl die Schauspieltradition als auch die Sozialhistorie Nürnbergs oder Berns gut untersucht sind. Was fehlt, ist der Brückenschlag zwischen religiös akzentuierter Bedrohungskommunikation und Sozialhistorie. Mit der Neucharakterisierung der Bedrohungskommunikation über das Konzept der >Bedrohten Ordnungen` scheint ein Ansatzpunkt gegeben, die Wechselseitigkeit von Sozialhistorie, Kommunikations- und Theatergeschichte neu aufzuschließen.

Der Artikel sucht daher Antworten auf folgende Fragen: (1) in welcher Weise nimmt medial-kommunikativer Wandel, also die mittels der neuen Drucktechnik vergrößerte Reichweite und Verfügbarkeit von Schauspieltexten, Einfluss auf die in den Bedrohungsszenarien dargestellten sozial wirksamen Prozesse, (2) inwiefern verweisen theologisch begründete Szenarien von Bedrohung auf soziale, wirtschaftliche und politische Transformationen. Die Schlusspassage fragt (3) danach, welche Typen von bedrohter Ordnung, von Bewältigungshandeln und von restituierter Ordnung in den analysierten Schauspielen dargestellt sind.

Hengartner, unter Mitarbeit von Barbara Freiburghaus. Bern 1999, S. 125-253. Zum Fastnachtspiel: Peter Pfrunder: Pfaffen, Ketzer, Totenfresser. Fastnachtskultur der Reformationszeit Die Berner Spiele von Niklaus Manuel. Zürich 1989, S. 189-240 (`Text und Kontext in Manuels Fastnachtspielen (); Glenn Ehrstine: Theater, Culture, and Community in Reformation Bern, 1523-1555. Leiden, Köln 2002, S. 79-117; Heidy Greco-Kaufmann: Niklaus Manuel, der Fastnachtspieldichter. In: Susan Marti (Hg.): Söldner, Bilderstürmer, Totentänzer. Mit Niklaus Manuel durch die Zeit der Reformation. Zürich 2016, S. 71-77; Klaus Ridder: Ordres menacés et innovations littéraires: les jeux carnavalesques de Niklaus Manuel pendant la Réforme. In: Fanny Platelle, Nora Viet (Hg.): Innovation - Révolution. Discours sur la nouveauté littéraire et artistique dans les pays germaniques. Clermont-Ferrand 2018, S. 37-54; Thomas Naogeorg: Sämtliche Werke. Hg. von Hans-Gert Roloff. Bd. 1: Dramen I: Tragoedia nova Pammachius nebst der deutschen Übersetzung von Johann Tyrolff. Berlin, New York 1975 (Ausgaben deutscher Literatur des XV. bis XVIII. Jahrhunderts). Zum Pammachius: Hans-Gert Roloff: Heilsgeschichte, Weltgeschichte und aktuelle Polemik: Thomas Naogeorgs Tragoedia Nova Pammachius. In: Hans-Gert Roloff: Kleine Schriften zur Literatur des 16. Jahrhunderts. Festgabe zum 70. Geburtstag. Hg. und eingeleitet von Christiane Caemmerer, Walter Delabar, Jörg Jungmayr, Wolfgang Neuber. Amsterdam, New York 2003, S. 230-357.

3 Eine performanz- und theatergeschichtliche Analyse von Bedrohungsszenarien ist, auf der Grundlage der drei hier herangezogenen Schauspiele, anderenorts erschienen: Klaus Ridder: Latenz und Aktualität. Bedrohungskommunikation im mittelalterlichen Schauspiel. In: Zeitschrift für deutsches Altertum und deutsche Literatur 149 (2020), S. 479-497. Zur Bedrohungskommunikation im deutschen und französischen Schauspiel vgl. auch Carlotta Posth: Bedrohungskommunikation im Theater des 14. bis 16. Jahrhunderts. Eine semiotische Untersuchung religiöser Schauspiele des deutschen und französischen Sprachraums. Mit einer diplomatischen Transkription des MS fr. 15063 der Bibliothèque nationale de France. Phil. Diss. Tübingen 2019. 


\section{Bedrohungsinszenierung in medial- kommunikationsgeschichtlicher Perspektive}

Die Konstitutions- und Wirkweise des Schauspiels lässt sich über den performativen Ansatz in besonderer Weise erschließen. Diese Perspektive stößt allerdings an Grenzen, wenn Zusammenhänge zwischen textuell-performativen Mustern in Darstellungsmedien (wie Schauspiel oder Predigt) und sozial-politischen Ordnungsmustern in den Blick kommen. Die Verschränkungen von medialer und sozialer Kommunikation hat vor allem Rudolf Schlögl methodisch als Form des Zugangs zur Transformation sozialer Strukturen und Institutionen konzeptualisiert. ${ }^{4}$ Auch die theatralen Bedrohungsszenarien lassen sich als Repräsentationen von sozialen Ordnungsmustern und politischen Wandlungsprozessen lesen; daher wird im Weiteren gefragt, ob Veränderungen der medial-äußeren Kommunikation (Aufführung, Handschrift, Druck) Einfluss auf die theatral-interne Kommunikation in den Stücken und auf die dargestellten Formen des Umgangs mit religiös-sozialer Verschiedenheit nehmen. ${ }^{5}$

\subsection{Kommunikation unter Anwesenden und über Distanzmedien}

Die drei Schauspiele entstehen und werden aufgeführt in einer Gesellschaft, deren soziale, religiöse und kulturelle Ordnungsmuster stark durch den Interak-

4 Schlögl vertritt einen »kommunikations- und medientheoretischen Ansatz [...], der es erlaubt, die vielfältigen und verzweigten Forschungen der Literaturwissenschaft und aller anderen historisch orientierten Textwissenschaften nicht nur als Ideengeschichte, sondern im genuinen Sinn als Beiträge zu einer Geschichte sozialer Ordnungsmuster zu lesen. Kulturwissenschaftliche Interdisziplinarität verlangt nach Methoden und Paradigmen, die den `Strom des Geistes` mit der Reproduktion des Sozialen historisch vermitteln. Dann kann man darauf verzichten so zu tun, als seien Menschen stets damit beschäftigt, soziale Strukturen oder Institutionen zu reproduzieren, weil Normen, Werte oder Sanktionsdrohungen ihnen dies nahelegen. « Rudolf Schlögel: Kommunikation und Vergesellschaftung unter Anwesenden. Formen des Sozialen und ihre Transformation in der Frühen Neuzeit. In: Geschichte und Gesellschaft: Zeitschrift für historische Sozialwissenschaft 34 (2008), S. 155-224, hier S. 158; vgl. auch Rudolf Schlögl: Politik beobachten. Öffentlichkeit und Medien in der Frühen Neuzeit. In: Zeitschrift für historische Forschung 35 (2008), S. 581-616.

5 »Insgesamt besteht natürlich die Gefahr, die strukturellen Grundlagen und materiell greifbaren, akuten Zuspitzungen einer Bedrohung in einem Untersuchungsdesign aus dem Blick zu verlieren, das Kommunikation zentral stellt. Doch hat jede Kommunikation einen Ermöglichungsraum, der kulturell und materiell beschrieben werden muss." Ewald Frie, Mischa Meier: Bedrohte Ordnungen. Gesellschaften unter Stress im Vergleich. In: Ewald Frie, Mischa Meier (Hg.): Aufruhr - Katastrophe - Konkurrenz - Zerfall (s. Anm. 1), S. 1-28, hier S. 23. 
tionstypus >Kommunikation unter Anwesenden` geprägt sind. Bereits in der Manuskriptkultur ab der Mitte des 15. Jahrhunderts, in besonderer Weise dann nach der Durchsetzung der neuen Drucktechnik in der ersten Hälfte des 16. Jahrhunderts, verändern Geschriebenes und Gedrucktes diese Form der Kommunikation und die mit ihr verbundenen sozialen Strukturgefüge. Die Drucktechnik befördert einen Gebrauch der Schrift, der kontextunabhängige Formen der Produktion und Verbreitung von Wissen und Sinnmustern etabliert: »Mit Schrift und Druck wuchsen der Kommunikation neue Reichweiten in Zeit und Raum zu «. ${ }^{6}$ Diese medialen und kommunikativen Transformationen nehmen Einfluss auf die Formen sozialer Ordnungsbildung. Sie gehen zudem einher mit Veränderungen literarischer oder theatraler Sinnbildungsprozesse.

Handschriftliche Schauspieltexte (aber etwa auch Predigten) sind im ausgehenden 15. und beginnenden 16. Jahrhundert dadurch gekennzeichnet, dass sie überwiegend in engem Zusammenhang mit Aufführungen entstehen und in einem begrenzten Kontext rezipiert werden. Sie sind in der Regel für die Träger (Redakteure, Regisseure, Schauspieler) solcher Aufführungen gedacht. ${ }^{7}$ Lesedramen, die

6 »Kommunikation über Printmedien entkoppelte Sender und Empfänger und ließ den Entstehungs- (wie auch den Empfangs-)Kontext einer Äußerung in Entfernung des Raumes und der Zeit verschwinden.« Schlögl: Politik beobachten (s. Anm. 4), S. 591.

7 Zur Typologie der handschriftlichen Überlieferung des geistlichen Schauspiels im deutschen Sprachraum Ursula Schulze: Geistliche Spiele im Mittelalter und in der Frühen Neuzeit. Von der liturgischen Feier zum Schauspiel. Eine Einführung. Berlin 2012, S. 21-33; Cornelia Herberichs: Lektüren des Performativen. Zur Medialität geistlicher Spiele des Mittelalters. In: Ingrid Kasten, Erika Fischer-Lichte (Hg.), Transformationen des Religiösen. Performativität und Textualität im geistlichen Spiel. Berlin, New York 2007, S. 167-185; Rolf Bergmann: Aufführungstext und Lesetext. Zur Funktion der Überlieferung des mittelalterlichen geistlichen deutschen Dramas. In: Herman Braet, Johan Nowé, Gilbert Tournoy (Hg.): The Theatre in the Middle Ages. Leuven 1985, S. 314-351; Hansjürgen Linke: Versuch über deutsche Handschriften mittelalterlicher Spiele. In: Volker Honemann, Nigel F. Pamer (Hg.): Deutsche Handschriften 1100-1400. Oxforder Kolloquium 1985. Tübingen 1988, S. 527-589.

$\mathrm{Zu}$ den Überlieferungstypen der französischen Tradition vgl. Cornelia Herberichs: Die »Zwieschlächtigkeit der Aufführung « und die »double diffusion« von Arnoul Grébans »Le Mystère de la Passion «. Anmerkungen zu Rainer Warnings Thesen zur Ambivalenz des Passionsspiels aus überlieferungsgeschichtlicher Perspektive. In: Jörn Bockmann, Regina Toepfer (Hg.): Ambivalenzen des Geistlichen Spiels. Revisionen von Texten und Methoden. Göttingen 2018, S. 195-219; Taku Kuroiwa, Xavier Leroux, Darwin Smith: De l'oral à l'oral: réflexions sur la transmission écrite des textes dramatiques au Moyen Âge. In: Médiévales 59 (2010), S. $17-$ 40; Darwin Smith: Les manuscrits » de thêâtre «. Introduction codicologique à des manuscrits qui n'existent pas. In: Gazette du livre médiéval 33 (1998), S. 1-10; Graham A. Runnalls: Towards a Typology of Medieval French Play Manuscripts. In: Graham A. Runnalls, Phillip E. Bennett (Hg.): The Editor and the Text. Essays in Honour of Anthony John Holden. Edinburgh 
sich an ein weiteres Publikum wenden, bleiben Ausnahmefälle. ${ }^{8}$ Gedruckte Spieltexte des 16. Jahrhunderts zielen demgegenüber auf einen größeren Adressatenkreis, ja sind tendenziell bereits für den deutschen Sprachraum verfasst. Sie liegen Aufführungen zugrunde, sind aber auch Lesetexte. ${ }^{9}$ Übersetzungen in europäische Volkssprachen weiten den Wirkungsraum der Spiel-Drucke noch einmal deutlich aus. Für die Verantwortlichen von Schauspiel-Aufführungen, die sich auf gedruckte Texte stützten, ergibt sich damit die Notwendigkeit, die Stücke in einen sozialen und kulturellen Rahmen einzupassen, der mit dem Entstehungskontext des Werkes zunehmend häufiger nicht mehr deckungsgleich ist. Dazu bedurfte es der Aktualisierung der Spieltexte, neuer performativer Darstellungstechniken und -konventionen sowie der Interpretation durch erläuternde Paratexte. Für das 16. Jahrhundert geht man insgesamt von einer Zunahme der Widmungsvorreden, Prologe und Epiloge aus, d. h. man vermutet eine Intensivierung der rezeptionssteuernden Elemente, die nicht Teil der Dramenhandlung sind und sich direkt an den Leser oder den Zuschauer einer Aufführung wenden. ${ }^{10}$

In der Überlieferung der untersuchten Schauspiele lässt sich diese Tendenz zur Ausweitung der Adressatenkreise (bzw. der Reduzierung der auf den Entstehungskontext verweisenden Elemente) und zur Vergrößerung der Reichweite durch in kurzen Abständen aufeinanderfolgende Druckauflagen deutlich ausmachen. Beim Herzog von Burgund, der zwischen 1486 und 1494 entstanden ist, stellt sich dies zunächst jedoch noch anders dar. Man kann mit großer Wahrscheinlichkeit von einer repräsentativen Aufführung des Fastnachtspiels in Nürnberg mit Billigung des Rats ausgehen. Überliefert ist das Stück nur in einer handschriftlichen Sammlung von Nürnberger Fastnachtspielen (und anderen Texten), die der Augsburger Kaufmann Claus Spaun geschrieben und zusammengestellt hat. ${ }^{11}$ Folz druckt einige seiner Fastnachtspiele (F 96 u. F 98 sind

1990, S. 96-113; Elisabeth Lalou, Darwin Smith: Pour une typologie des manuscrits de théâtre médiéval. In: Fifteenth-Century Studies 13 (1988), S. 569-579.

8 Dies gilt vermutlich nicht in gleicher Weise für Schauspiele in lateinischer Sprache.

$9 \mathrm{Zu}$ Dramen(-texten) unter den kommunikativen Bedingungen des Buchdrucks vgl. Julie Stone Peters: Theatre of the Book, 1480-1880: Print, Text, and Performance in Europe. Oxford 2000; Graham A. Runnalls: Les mystères français imprimés: une étude sur les rapports entre le théâtre religieux et l'imprimerie à la fin du Moyen Age français. Suivi d'un Répertoire complet des mystères français imprimés (ouvrages, édition, exemplaire), 1484-1630. Paris 1999.

10 Vgl. Werner Wolf: Prologe als Paratexte und/oder dramatische (Eingangs-)Rahmungen? >Literarische Rahmung als Alternative zum problematischen Paratext-Konzept. In: Frieder von Ammon, Herfried Vögel (Hg.): Die Pluralisierung des Paratexts in der Frühen Neuzeit. Theorie, Formen, Funktionen. Berlin 2008, S. 79-98.

11 Wolfenbüttel, Herzog-August-Bibliothek, Cod. Guelf. 18.12. Aug. $4^{\circ}$, fol. 109v-124r. Eine Autorsignatur fehlt dem Herzog von Burgund. Christoph Gerhardt sieht dies u. a. darin begründet, 
erhalten) in der eigenen Druckerei, nicht jedoch den Herzog von Burgund. Man entscheidet sich also bewusst gegen eine druckschriftliche Verbreitung des Stücks, als die neue Technik in der Stadt zur Verfügung stand und dem Verfasser das neue Medium durchaus vertraut war. ${ }^{12}$ Dieser Präferierung von Verbreitungsmedien, die sich auf eine lokale Situation ausrichten, entspricht die kommunikative Konstellation, die das Stück gestaltet. Obwohl dies nicht explizit wird, ist doch deutlich, dass das Handlungsgeschehen (der Herzog von Burgund besucht die Stadt zur Fastnacht) und die evozierte Bedrohungssituation (die Aufdeckung einer Verschwörung der Juden) in Nürnberg zu denken sind.

Das Fastnachtspiel Niklaus Manuels Vom Papst und seiner Priesterschaft wurde 1523 in Bern aufgeführt. Es gibt eine fragmentarische Handschrift des Stücks, die wohl in der Zeit »zwischen der Aufführung und dem ersten Druck « ${ }^{13}$ entstanden ist. Das Spiel wird dann, vermutlich in der Druckerei von Christoph Froschauer in Zürich (in Bern existiert bis 1537 keine Offizin), in rascher Folge mehrfach gedruckt: im Mai 1524, im August 1524, ein weiterer Druck im Jahr 1524 sowie im Januar 1525. ${ }^{14}$ Der Berner Chronist Valerius Anshelm schreibt, dass die Aufführungen der Spiele in Bern großes Interesse fanden und dass darüber hinaus kein anderes Buch in den Auseinandersetzungen um den evangelischen Glauben so häufig gedruckt und so weit verbreitet worden sei. ${ }^{15}$ Die

dass die »Aufzeichnungen« Spauns, »im Gegensatz zu dem ursprünglichen `Sitz im Leben ১der Spiele selbst, privat, nicht für eine breitere Leseöffentlichkeit oder gar für eine Aufführung in der Öffentlichkeit vor einem `Theater`-Publikum gedacht waren«; eine Autorsignatur dürfte daher »für Spaun längst nicht eine solche Bedeutung gehabt haben, wie sie sie u. U. für die Verantwortlichkeit des Autors gegenüber dem auch die schriftlichen Aktivitäten nicht nur der Fastnacht kontrollierenden Nürnberger Rat hatte«; Christoph Gerhardt: Hans Folz, der Berner und der Wunderer. In: Martin Przybilski (Hg.): Studien zu ausgewählten Fastnachtspielen des Hans Folz. Struktur - Autorschaft - Quellen. Wiesbaden 2011, S. 37-97, hier S. 40.

12 Eine eigene Offizin betrieb Hans Folz von 1479 bis 1488; danach lässt er bei Peter Wagner und Hans Mair in Nürnberg drucken; zur Drucker- und Verlagstätigkeit des Autors vgl. Caroline Huey: Hans Folz and Print Culture in Late Medieval Germany. The creation of popular discourse. Farnham, Burlington 2012; John L. Flood: Hans Folz zwischen Handschriftenkultur und Buchdruckerkunst. In: Elizabeth Andersen, Manfred Eikelmann, Anne Simon (Hg.): Texttyp und Textproduktion in der deutschen Literatur des Mittelalters. Berlin, Boston 2005, S. 1-27; Ursula Rautenberg: Das Werk als Ware. Der Nürnberger Kleindrucker Hans Folz. In: IASL 24 (1999), S. 1-40.

13 Deutsche Spiele und Dramen des 15. und 16. Jahrhunderts. Hg. von Hellmut Thomke. Frankfurt a.M. 1996, S. 999.

14 Auflistung der Drucke in Manuel: Werke und Briefe (s. Anm. 2), S. 116-124.

15 "Es ist ouch in dem evangelischen handel kum ein bueechle so dik getrukt und so wit gebracht worden, als diser spilen «. Die Berner-Chronik des Valerius Anshelm. Hg. vom Historischen Verein des Kantons Bern. Bern 1893, Bd. 4, S. 475. 
Verschränkung von alten und neuen, von Medien unterschiedlicher Reichweite verschiebt sich hier deutlich in Richtung einer Präferenz des Distanzmediums Buchdruck.

Die interne Kommunikationssituation des Stücks ist geprägt durch das gespannte Gegenüber von Repräsentanten des neuen und des alten Glaubens: Neben den Funktionsträgern der alten Kirche, werden Bauern-Figuren aus dem eidgenössischen Raum einbezogen, am päpstlichen Hof ist die Begegnung zwischen Kreuzritter und Papst zu denken, im Himmel der Dialog zwischen den Aposteln Petrus und Paulus. Der Raum, in dem das Stück wirken will, nämlich die Stadt Bern, bleibt weitgehend ausgespart. In dem Maße wie der explizite Bezug auf einen städtischen Raum zurückgenommen wird, eröffnet sich die Möglichkeit zu weiteren Aufführungen in Städten und Territorien der Eidgenossenschaft, in denen das protestantische Anliegen bereits einen gewissen Rückhalt hatte.

Am Pammachius des Thomas Naogeorg wird deutlich, wie das Schauspiel unter den kommunikativen Bedingungen der sich rasch entwickelnden Drucktechnik und der europäischen Übersetzungskultur dazu beiträgt, »daß aus den verschiedenen städtischen Einzelgeschehen `dieく Reformation als überlokaler Ereignis- und Erfahrungszusammenhang werden konnte«. Dabei ist jedoch der »Anteil der [...] performativen Ereignisse der Anwesenheitskommunikation ${ }^{16}$ etwa durch Schauspielaufführungen, die Fragen des Heils, der sozialen Ordnung oder der göttlichen Weltordnung tangierten - nicht zu unterschätzen. Der Erstdruck des Stücks bei Johannes Luft in Wittenberg in lateinischer Sprache datiert auf den 13. Mai 1538. Es gibt keinen Nachweis dafür, dass das Stück auch in Wittenberg aufgeführt worden ist. 1539 und 1540 erscheinen zwei weitere Auflagen des lateinischen Textes. In diesen beiden Jahren folgen auch vier deutsche Übersetzungen mit umfangreichen Begleittexten an verschiedenen Orten in Deutschland; die Übersetzung von Hans Tyrolff (1540) wurde von Naogeorg autorisiert. Bereits 1546 wird das Stück dann ins Tschechische übersetzt und vermutlich schon 1538/39 geht eine (nicht erhaltene) englische Übersetzung von John Bale voraus. ${ }^{17}$

16 Schlögl: Politik beobachten (s. Anm. 4), S. 606.

17 Zur Druck- und Übersetzungsgeschichte des Werkes vgl. Naogeorg: Pammachius (s. Anm. 2), Nachwort des Herausgebers, S. 594-619; Roloff: Heilsgeschichte (s. Anm. 2), S. 344. - Die Übersetzung des Pammachius ins Englische wurde eventuell von Thomas Cranmer (Reformation Archbishop of Canterbury) oder Thomas Cromwell (viceregent for religion) in Auftrag gegeben, die beide im Dienst Henrys VIII. standen. Rückschlüsse auf die Rezeption des Stückes in England lassen sich anhand der Zeugnisse einer öffentlichen Aufführung (vermutlich in lateinischer Sprache) am Christ's College, Cambridge, im Jahr 1545 ziehen. Trotz der zensierten Sprechtexte hatte das Schau- 
Der Pammachius wendet sich an einen überregionalen, der lateinischen Sprache mächtigen, also tendenziell europäischen Adressatenkreis. Die Übersetzungen zunächst ins Deutsche, dann in weitere europäische Volkssprachen verstärken die sraumübergreifendeく Rezeption des Stücks. Mit Ausnahme der Angabe des Druckortes finden sich keine Elemente, die auf einen lokalen Entstehungs- oder Aufführungskontext verweisen. Die späteren Übersetzer fügen jedoch zum Teil umfangreiche Widmungen und Vorreden, Erläuterungen und Positionierungen, hinzu, in denen auch mediale und soziale Kommunikation zum Thema wird. ${ }^{18}$ Naogeorg begreift im Pammachius die Reformation als eine transregionale, wenn nicht gar als eine europäische Bewegung. Die Perspektive des Handlungsgeschehens ist eine globale, die Reformation ist als ein Ereignis von heilsgeschichtlich-welthistorischer Bedeutung dargestellt. Die Veränderung der kommunikativen Bedingungen, unter denen das Stück entstanden ist, nimmt auch Einfluss auf die in ihm ausgeformten Bedrohungsszenarien: diese sind nicht mehr auf den Horizont einer identifizierbaren Stadtgesellschaft, sondern auf Anhänger der protestantischen Lehre in größeren konfessionell geprägten Räumen ausgerichtet.

\subsection{Radikale Exklusion und räumliche Ausdifferenzierung von religiöser Verschiedenheit}

Man kann fragen, welchen Einfluss die Veränderungen der medialen und sozialen Kommunikation auf die Formen des Umgangs mit religiös-sozialer Verschie-

spiel eine stark polarisierende Wirkung: Katholische Zuschauer empfanden die Aufführung als Provokation, radikale Anhänger der reformatorischen Ideen mobilisierte die Schlussszene zu Ausschreitungen gegenüber Altgläubigen; dazu Paul Whitfield White: The Pammachius Affair at Christ's College, Cambridge, in 1545. In: Peter Happé, Wim Hüsken (Hg.): Interludes and Early Modern Society. Studies in Gender, Power and Theatricality. Leiden 2007, S. 261-290.

Eine französische Übersetzung des Pammachius hat es wohl nicht gegeben; gleichwohl rezipiert man Naogeorgs Ideen im französischen Sprachraum über eine Zwischenstufe: Der 1556 in Basel erschienene Christus Triumphans (lat.) des John Foxe lässt deutlich den Einfluss des Pammachius erkennen; vgl. John Hazel Smith (Hg.): Two latin comedies by John Foxe the Martyrologist: Titus et Gesippus; Christus Triumphans. Ed. with Introduction, Translation, and Notes by John Hazel Smith. Ithaca, London 1973, Introduction S. 44.1562 publiziert dann Jacques Bienvenu in Genf Le Triomphe de Jesus Christ, eine übersetzende Bearbeitung des Stücks von John Foxe; dazu Charlotte Bouteille-Meister: La traduction française du Christus Triumphans, comœdia apocalyptica de John Foxe (Genève, 1562): une (re)lecture biblique de l'actualité des conflits religieux. In: Véronique Ferrer, Jean-René Valette (Hg.): Écrire la Bible en français au Moyen-Age et à la Renaissance. Genf 2017, S. 703-721.

18 Vgl. Naogeorg: Pammachius (s. Anm. 2), Anhang I-V, S. 477-585. 
denheit nehmen. Kommunikation unter Anwesenden ist sehr effektiv, vermag enormen Konsensdruck zu erzeugen, ist jedoch weniger pluralistisch und deshalb konfliktgefährdet. Diese Form der Kommunikation fördert nicht unbedingt, so Rudolf Schlögl, »die Fähigkeit der Gesellschaft, Verschiedenheit zu integrieren«. Die sozialen Einheiten reagieren auf Differenz häufig durch öffentliche Degradation und soziale Exklusion. Das Distanzmedium Druck-Schrift »entlastete [demgegenüber] offenbar vom Aufmerksamkeits- und Konsensdruck der Anwesenheitskommunikation . $^{19}$

Betrachtet man die Figurationen der Bedrohungsszenarien in den Spieltexten, die in einem Zeitraum von etwa 55 Jahren entstehen, so lässt sich erkennen, dass sich die Relation von Inklusion und Exklusion von Personen mit abweichenden religiösen Auffassungen verändert. ${ }^{20}$ Im Nürnberger Fastnachtspiel werden den Juden gravierende Verstöße gegen das Gemeinwohl vorgeworfen, so dass man ihnen jede weitere Kommunikation und das Recht auf Verbleib im Sozialverbund verweigert. Sie werden Objekte herabsetzender und entehrender sozialer Praktiken und körperbezogener Strafgewalt. Bezieht man den von der historischen Forschung analysierten Kontext der Ausweisung der Juden von 1498/99 in der Stadt hinzu, so wird auf eine wirtschaftliche Veränderungsdynamik mit Restriktion und Ausgrenzung reagiert.

Das Fastnachtspiel Manuels will die Durchsetzung der Reformation insbesondere in der städtischen Führungsschicht befördern, zielt jedoch nicht auf Bestrafung oder Exklusion der Anhänger des alten Glaubens aus der sozialen Gemeinschaft. Die politische Konsequenz der Forderung, die Funktionsträger der alten Kirche zu entmachten, bleibt ausgespart. Die fehlende Orientierung des Handelns der religiösen Amtsträger am gemeinen Nutzen aufzuzeigen, ist ein zentrales Anliegen des Stücks. Amtsenthebung und Entmachtung sind die angemessene Reaktion auf dieses Vergehen, nicht jedoch kollektiver Ausschluss, soziale Ächtung oder gravierende Bestrafung. Man macht einen Unterschied zwischen Juden und (Mit-)Christen; in nachfolgenden Stücken intensiviert sich jedoch das Moment der Verfolgung von konfessionell Andersgläubigen. ${ }^{21}$

19 »Eine Gesellschaft, die sich auf die expandierende Nutzung der Drucktechnik einließ, mußte daher lernen, mit der Pluralität der Ansichten zu leben, und sie mußte Strategien entwickeln (vorzugsweise kommunikative), um kommunikative Anschlüsse unter solch schwierigen Bedingungen sicherzustellen. « Schlögl: Politik beobachten (s. Anm. 4), S. 585 und S. 592.

20 Zur Entwicklung der Verweisungsstrafen im städtischen Kontext vgl. Gerd Schwerhoff: Vertreibung als Strafe. Der Stadt- und Landesverweis im Ancien Régime. In: Sylvia Hahn, Andrea Komlosy, Ilse Reiter (Hg.): Ausweisung - Abschiebung - Vertreibung in Europa. 16.-20. Jahrhundert. Innsbruck, Wien, Bozen 2006, S. 48-72.

21 Dazu Klaus Ridder: La persécution comme menace dans le théâtre de la Réforme (15251538). In: Revue d'Histoire du Théâtre nº 286 (2020), S. 95-106. 
Der Pammachius inszeniert den konfessionellen Konflikt als globales Ereignis: Weltweit sind nur noch 7000 Christen übrig geblieben, die den Verlockungen der Teufels- und Papstherrschaft (noch) nicht verfallen sind. In diesem Moment bricht in Wittenberg die Reformation aus und der teuflische Papst (Pammachius) aktiviert weltliche und geistliche Machthaber, um die Ketzer mit Heeresmacht zu vernichten (vv. lat. 3299-3301; dt. 4375-4378). Die Kontrastierung einer kleinen Zahl von Rechtgläubigen mit einem großen Aufgebot der Teufelsherrschaft projiziert das Gegenüber von bereits konfessionell geprägten größeren Räumen, also die Situation zur Zeit Naogeorgs, auf den Beginn der Reformation. Die Perspektive der Ausmerzung von als Häretiker identifizierten Individuen, also der Blick der alten Kirche etwa auf Personen wie Jan Hus, wird auf die konfessionelle Sozialgemeinschaft übertragen. Beide Zuspitzungen weisen darauf hin, dass eine Ausdifferenzierung konfessioneller Räume im Gange ist, dass die radikale Auslöschung der Anhänger der Reformation keine ernstzunehmende Option mehr ist, dass dem im Stück als Bedrohung inszenierten HäresieKonzept bereits ein Denken in den Bahnen religiöser Devianz bzw. Verschiedenheit gegenübersteht.

Die vorausgehenden Überlegungen, so lässt sich festhalten, gingen der Frage nach, inwiefern die sozialen Formen des Umgangs mit religiöser Anders- oder Verschiedenheit durch den medialen Umbruch in der zweiten Hälfte des 15. und im frühen 16. Jahrhundert verändert wurden. Der These einer dynamischen Veränderung dieser Relation durch das Zusammenspiel von neuen medialen Möglichkeiten und neuen religiösen Ideen in der Reformationszeit ist jedoch die einer allmählichen Anbahnung von Veränderungsprozessen bereits im 15. Jahrhundert und deren Intensivierung durch die Reformation zur Seite zu stellen. Für Guy Borgnet beispielsweise hat die Verschränkung von religiös-politischer Thematik und antijüdisch-aggressiver Polemik in den Fastnachtspielen des Hans Folz dem polemischen Reformationstheater den Weg bereitet. ${ }^{22}$ Die Ausformung der innerchristlichen Polemik erreicht mit der Reformation sicher ihren Höhepunkt, ebenso wie die Praxis der religiös-politischen Verfolgung auch im konfessionellen Zeitalter ein wichtiges Element sozialer Strukturbildung bleibt.

22 »En introduisant les thèmes religieux et la polémique antijuive dans ses jeux, il ne fait pas de doute que Folz ait cherché à faire éclater le genre, à le faire sortir des sentiers battus. II en fait à la fois un jeu religieux et un jeu politique. Avec Folz, le Jeu de Carnaval devient une arme de propagande et se met au service d'une cause. Par là, Folz annonce les transformations du seizième siècle. Il prépare le théâtre polémique qui verra le jour au moment de la Reforme." Guy Borgnet: Jeu de Carnaval et Antisémitisme: L'intolérance chez Hans Folz. In: Études médiévales 5 (2003), S. 1-9, hier S. 9. 


\section{Bedrohungskommunikation in theologie- sozialgeschichtlicher Perspektive}

Wie ist nun der Bogen von den religiös akzentuierten Bedrohungsszenarien in den Schauspielen zur sozial-politischen Ordnung zu schlagen? Die auf konkrete historische Situationen zugeschnittenen Bedrohungsszenarien in den Schauspielen sind auch Ausdrucksformen und Deutungen übergreifender sozialer, ökonomischer und politischer Wandlungsprozesse der Gesellschaft. Solche Wandlungsprozesse vollziehen sich längerfristig, zeichnen sich aber in Situationen akuter bedrohter Ordnung ab, haben sich jedoch noch nicht in breiter Fläche durchgesetzt. Religiöse Bedrohungsszenarien lenken so den Blick auf latente und aktuelle Spannungsfelder sozialen und ökonomischen Wandels.

Die religiösen Szenarien erzählen von Bedrohung, von Veränderung, von Neukonstituierung; sie sind damit bedeutsam für die Erklärung geschichtlichen Wandels. Die sozialen, ökonomischen und politischen Prozesse entziehen sich der direkten Beschreibung; sie sind nur auf indirekte Weise (über Deutung, Interpretation) wahrnehmbar. Auf der Ebene der theatralen Repräsentation haben sie also Latenzstatus. Auch auf der Ebene historischer Realität sind sie in der >normalen s sozialen Kommunikation sicher kaum präsent. In Situationen bedrohter oder zerbrechender Ordnung können sie jedoch Aktualitätsstatus gewinnen; sie werden beobachtbar. Die Konstruktion und Aufführung von Bedrohungsszenarien macht die Verweiszusammenhänge zwischen religiösen und sozialen sowie zwischen aktuellen und latenten Wandlungsprozessen einsehbar. Einige Bemerkungen zu den untersuchten Spielen können diese Perspektive verdeutlichen.

\subsection{Kirchliches Zinswucherverbot und kapitalorientierte Wirtschaftspraktiken}

Die Inszenierung des Bedrohungsszenariums Jüdische Verschwörung, dem man durch den definitiven Ausschluss der Juden aus der Kommune begegnet, ist nicht nur durch den Faktor der >falschen Religion motiviert. Sowohl politische als auch ökonomische Gründe sind als treibende Kräfte hinter Ausweisungen von jüdischen Gemeinden aus zahlreichen Städten im europäischen Raum des 15. Jahrhunderts erkennbar. Schon im Pogrom von 1349 ging es dem Nürnberger Rat darum, sich in Besitz des im Zentrum der Stadt gelegenen Judenviertels zu bringen. Die Beschlagnahme jüdischer Immobilien ist dann auch ein Beweg- 
grund für die Vertreibung von $1498 .^{23}$ Dass wirtschaftliche Gründe für das Handeln der an Pogromen und Austreibungen beteiligten Christen eine wichtige Rolle gespielt haben, gilt der Forschung inzwischen als gesichert. ${ }^{24}$ Formen konkreter Beraubung symbolisiert das Fastnachtspiel des Hans Folz dadurch, dass man den Juden ihre Börsen abnimmt, bevor sie aus der Stadt getrieben werden. Wenn der falsche Messias bekennt, dass die Juden den Christen seit langer Zeit große Güter entzogen ([...] vil groß guts in abgeraubt, v. 356) hätten, dann verweist dies insbesondere auf jüdische Geldgeschäfte, die zwar in Nürnberg im 15. Jahrhundert stark an Bedeutung verloren hatten, die jedoch traditionell als Kernbereich jüdischer Erwerbsformen gesehen wurden. Der größte Teil der Einwohner Nürnbergs, die Handwerkerschaft, wird in der Aussage des Stücks eine Antwort auf die Frage nach den Ursachen ihrer zunehmenden Verarmung in der zweiten Hälfte des 15. Jahrhunderts gesehen haben. In seiner Legitimation der Austreibung rekurriert auch der Rat ausdrücklich darauf, die Bürger vor den Folgen der jüdischen Geld- und Pfandleihe schützen zu wollen. Das Bedrohungsszenarium Jüdische Verschwörung und ebenso die Erklärungen des Rates geben eine (unzutreffende) Antwort auf einen bedeutenden ökonomischen und sozialen Wandlungsprozess, der in den großen süddeutschen Reichsstädten im 15. Jahrhundert in vollem Gange ist: der stetige

Aufstieg [...] eines nichtpatrizischen Großbürgertums von Finanzmaklern, Gewerbeverlegern, Montanunternehmern und Großkaufleuten, die gewichtige Teile der Nürnberger Handwerksberufe in Abhängigkeit brachten.

Der Rat hatte sich in dieser Zeit mit sozio-ökonomischen Veränderungen auseinanderzusetzen, »die einerseits ein neues Großbürgertum schufen und andererseits das zahlenmäßig bedeutende Handwerkertum, das eigentliche Stadtvolk, zu entwurzeln drohten «. ${ }^{25}$ Indirekt verweist das latent-traditionelle Bedrohungsszenarium Jüdische Verschwörung damit auf einer zweiten, aktuellen Ebene auf einen sozio-ökonomischen Strukturwandel, der erhebliche Teile der Stadtbevölkerung pauperisierte. Zwei Tendenzen einer überregionalen Entwicklung werden hier sichtbar: Auf der einen Seite evoziert die Expansion von frühkapitalistischen

23 Dazu Michael Toch: »umb gemeyns nutz und nottdurfft willen«. In: Zeitschrift für historische Forschung 11-1/4 (1984), S. 1-21, hier S. 5f. sowie S. 18 (Text des Austreibungsmandats von König Maximilian I.).

24 Zu diesem Aspekt vgl. Christian Scholl: Judenfeindschaft in den deutschen Städten des Mittelalters: Die Bedeutung des Faktors Religion. In: Jörg Oberste (Hg.): Pluralität - Konkurrenz - Konflikt. Religiöse Spannungen im städtischen Raum der Vormoderne. Regensburg 2013, S. 175-188, insbesondere S. 178 f., $182 \mathrm{f}$.

25 Toch: »umb gemeyns nutz und nottdurfft willen« (s. Anm. 23), S. 8 f. (beide Zitate). 
Wirtschaftsformen und kapitalorientierten Praktiken in Spätmittelalter und Früher Neuzeit Wucher-, Habgier- und antijüdische Debatten. Auf der anderen Seite entkoppelt sich wirtschaftliches Handeln von einschränkenden religiösen Normen, also vor allem vom kirchlichen Zinswucherverbot. Gewinnstreben und Effizienz gelten nicht mehr nur als individuelle Laster, sondern zunehmend als zentrale Tugenden ökonomischen Verhaltens in Wirtschaft und Handel. ${ }^{26}$

\subsection{Altgläubige Jenseitsvorsorge und reformatorisches Gemeinwohl}

In der einleitenden Totenfresser-Szene (Papst und Klerus beim Festmahl anlässlich einer Beerdigung) rückt Niklaus Manuel das System der durch die Gläubigen zu erbringenden Kirchenopfer (z. B. für Toten- und Seelenmessen) als eine Art finanzieller Ratenzahlung für im Jenseits zu erwartendes Seelenheil in den Blick. ${ }^{27}$ Die vehemente Polemik gegen diese unerträgliche Form der Bereicherung von kirchlichen Funktionsträgern bringt auch die politischen Verhältnisse Berns vor der Reformation in die Debatte. Indem es der Herrschaftselite den Glaubenswechsel nahelegt, fordert das Fastnachtspiel Manuels tiefgreifende religiöse Umwälzungen ein, die erkennbar auch soziale und politische Veränderungen nach sich ziehen würden. Dabei ist nicht nur an den Aufstieg von neuen Familien in die städtische Führungsschicht bzw. den Abstieg von etablierten Familien zu

26 Die Kirche hat zwar sehr lange am Wucherverbot festgehalten, doch ließ man Zinserträge unter bestimmten Bedingungen durchaus zu. Daher ging die Forschung lange »von einer latenten Diskrepanz zwischen Ethik und Finanzgebaren aus«. Inzwischen hat sich das Erkenntnisinteresse auf die Untersuchung einer sozial »eingebetteten, vormodernen Kredit- und Geldkultur« verlagert. So ist beispielsweise auch zeitgenössischen Theologen nicht verborgen geblieben, »dass eine Wertschöpfung der Sozialorganisation nicht zuwiderlief, sondern in einem förderlichen Verhältnis zu ihr stand. Relativ zügig wurde demzufolge unterschieden zwischen dem infamen Wucher unproduktiver Subjekte, deren alleinige Tätigkeit im professionellen Geldverleih bestand, und einer gerechtfertigten Zinsnahme [...] öffentlich anerkannter Akteure.« Julia Zunckel: Die Kontroverse um die Genueser Wechselmessen im Pontifikat Gregors XV. Wucher, Kredit und Kommerz im Zeichen der Normenkonkurrenz. In: Arne Karsten, Hillard von Thiessen (Hg.): Normenkonkurrenz in historischer Perspektive. Berlin 2015, S. 141-169, hier S. 164, 155.

27 Übergreifend Berndt Hamm: Den Himmel kaufen. Heilskommerzielle Perspektiven des 14. bis 16. Jahrhunderts. In: Ders. (Hg.): Religiosität im späten Mittelalter. Spannungspole, Neuaufbrüche, Normierungen. Tübingen 2011, S. 301-334. Zum >Totenkult als Kampfzone der beginnenden Reformation « in Bern vgl. Uwe W. Dörk: Totenkult und Geschichtsschreibung. Eine Konstellationsgeschichte zwischen Mittelalter und Moderne (Bern und Ulm). Konstanz 2014, S. 109-163 (Analyse der Kritik am ökonomisierten Totenkult in den Werken Niklaus Manuels). 
denken. Die Infragestellung der für die individuelle Heilssicherung aufzuwendenden Zahlungen der Gläubigen lenkt das Augenmerk auch auf das System der Pensionen, Miet und Gaben an die Mitglieder der Berner Führungsschicht.

Bei den Pensionen handelte es sich um Zuwendungen der europäischen Potentaten an Orte der Eidgenossenschaft, um sich das Recht zu sichern, Reisläufer (Söldner) anzuwerben. Teilweise gehen die Pensionsgelder im Verborgenen auch an einzelne Repräsentanten der Berner Führungsgruppe. Zumindest diese Zuwendungen haben den Charakter von »unerlaubte[n] [Geld-]Geschenke[n] an Amtspersonen ${ }^{28}{ }^{28}$ Bereits seit dem ausgehenden 15. Jahrhundert diskutiert man diese Praxis unter dem Gesichtspunkt der guten und gerechten Herrschaft. Im Berner Reformationsmandat von 1528 wird dann die Abschaffung dieser Zahlungen verkündet, weil sie `fremde Kriege`, Zwietracht und Zerrüttung beförderten. Zwingli brachte den Kampf gegen die Korruption mit der konfessionellen Problematik in Zusammenhang. Er brandmarkte die Altgläubigen über die Begriffe Pensionen, Miet und Gaben (»als bestechlich, abhängig von auswärtigen Mächten und als Verräter an der Eidgenossenschaft « ${ }^{29}$ ) als Gegner von Evangelium und Gemeinwohl. Die ältere Debatte um die Pensionen wandelte sich in der Reformationszeit zu einer Diskussion über das Verhältnis von Eigennutz und Gemeinnutz. Bei der Verkündigung des Reformationsmandats stellt das

28 Florian Schmitz: Normenkonkurrenz oder Normenkongruenz? Dimensionen eines Konzeptes anhand eines Korruptionsfalles von 1534. In: Normenkonkurrenz in historischer Perspektive (s. Anm. 26), S. 65-79, hier S. 70; zur Diskussion über die Pensionen demnächst Maud Harivel, Florian Schmitz, Simona Slanicka (Hg.): Fremde Gelder? Pensionen in der frühneuzeitlichen Eidgenossenschaft (für 12/2021 angekündigt). Man erwartete politische und religiöse Patronage von Persönlichkeiten mit Einfluss; bei Amtsinhabern kollidierte die verbreitete soziale Praxis jedoch mit gemeinwohlorientierten Normen. Dieselben Handlungen konnten daher unterschiedlich bewertet werden; zur politischen Korruptionsforschung vgl. Niels Grüne: »Und sie wissen nicht, was es ist «. Ansätze und Blickpunkte historischer Korruptionsforschung. In: Niels Grüne, Simona Slanicka (Hg.): Korruption. Historische Annäherungen an eine Grundfigur politischer Kommunikation. Göttingen 2010, S. 11-34.

29 Valentin Groebner: Gefährliche Geschenke. Ritual, Politik und die Sprache der Korruption in der Eidgenossenschaft im späten Mittelalter und am Beginn der Neuzeit. Konstanz 2000, S. 245; zum Spannungsfeld von Gemeinnutz und Eigennutz vgl. dort S. 185 f.; 236f., 263. »Eigen$n u t z$ wurde von den Theologen der alten Kirche - dem Papst, den Bischöfen, den Mönchen vorgeworfen, aber nicht nur ihr, sondern allen, die sich gegen einen christlichen Lebenswandel, das war jetzt gleichbedeutend mit einem Bekenntnis zur Reformation, sperrten. Eigennutz ist gottlos, gehört zu den ständig memorierten und repetierten Aussagen von Huldrich Zwingli, folglich dient derjenige dem Gemeinen Nutzen, der sich praktisch zum reformatorischen reinen Evangelium bekennt.« Peter Blickle: Der Gemeine Nutzen. Ein kommunaler Wert und seine politische Karriere. In: Herfried Münkler, Harald Bluhm (Hg.): Gemeinwohl und Gemeinsinn. Historische Semantiken politischer Leitbegriffe. Berlin 2001, S. 85-197, hier S. 103. 
Berner Stadtregiment den Kampf gegen die Korruption als eine unverzichtbare Verpflichtung der Obrigkeit im Sinne des Gemeinwohls dar. Offenbar war man bemüht, diese Forderung auch umzusetzen, zumindest versucht man ihr 1534 juristisch Nachdruck zu verleihen. In diesem Jahr werden vier Patrizier in Bern vor diesem Hintergrund der Bestechlichkeit angeklagt. Der Berner Stadtchronist Valerius Anshelm betrachtet diesen exemplarischen Prozess als »Teil des reformatorischen Reinigungsprozesses des städtischen Gemeinwesens « ${ }^{30}$.

So selbstverständlich politische Patronage als soziale Praxis in der Stadtgesellschaft ist und bleibt, so eröffnet erst das mit ihr verknüpfte Gegenbild der Korruption einen abwägenden Blick auf gemeinwohlorientierte Handlungsweisen und -erwartungen im Raum des Politischen. In der reformatorischen Kritik an den religiösen Praktiken einer individuellen und materiellen Heilsvorsorge ist eine kritische Perspektive auf die sozialen und politischen Praktiken des Machterwerbs und des Machterhalts in der städtischen Gemeinschaft mitzudenken. Die Denkform von der Kommune als Heilsgemeinschaft ${ }^{31}$ harmonisiert auf der einen Seite religiöse und gemeinwohlorientierte Normen, vermag aber auch die Wahrnehmung zu schärfen für zu enge und zu selbstverständliche Verflechtungen des Sozialen, des Politischen und des Religiösen.

\subsection{Religiöse Devianz und politischer Neuanfang}

Das Pammachius-Drama des Naogeorg bringt Welt- und Heilsgeschichte als teleologisch-apokalyptisches Geschehen auf die Bühne. Die Situation religiöspolitischer Verfolgung von Andersdenkenden bildet die Leitperspektive der Darstellung: ${ }^{32}$ In römisch-frühchristlicher, christlich-papistischer und auch in christ-

30 Dazu der Aufsatz von Schmitz: Normenkonkurrenz (s. Anm. 28); Zitat S. 77.

31 Zur Auseinandersetzung mit der Idee einer städtischen `Sakralgemeinschaft ‘ vgl. Thomas Kaufmann: Einleitung. In: Bernd Moeller: Reichsstadt und Reformation. Neue Ausgabe. Hg. von Thomas Kaufmann. Tübingen 2011, S. 1-38, hier S. 25; Alexander Kästner, Gerd Schwerhoff: Religiöse Devianz in alteuropäischen Stadtgesellschaften. Eine Einführung in systematischer Absicht. In: Alexander Kästner, Gerd Schwerhoff (Hg.): Göttlicher Zorn und menschliches Maß: Religiöse Abweichung in frühneuzeitlichen Stadtgemeinschaften. Konstanz, München 2013, S. 9-43, hier S. 15-17.

32 Zum Problemfeld `Religiöse Devianz` in Spätmittelalter und Früher Neuzeit vgl. die Forschungsberichte von Alexander Kästner, Gerd Schwerhoff: Religiöse Devianz in alteuropäischen Stadtgesellschaften (s. Anm. 31), S. 1-38; Eric Piltz, Gerd Schwerhoff: Religiöse Devianz im konfessionellen Zeitalter - Dimensionen eines Forschungsfeldes. In: Eric Piltz, Gerd Schwerhoff (Hg.): Gottlosigkeit und Eigensinn. Religiöse Devianz im konfessionellen Zeitalter. Berlin 2015, S. 9-50. 
lich-evangelischer Zeit ist diese Erfahrung als latente Bedrohung präsent. Diese dramatische Strukturierung von Geschichte im Pammachius erinnert an entmythologisierte Formen des Weltalterdenkens. Die Handlung setzt im ersten Akt unmittelbar nach dem Ende der Christenverfolgung und der Erhebung des Christentums zur Staatsreligion ein. In der sich anschließenden Niedergangs- und Verfallsphase unterwirft der Papst, im Verein mit dem Teufel, die weltliche Macht und übernimmt die Weltherrschaft - ein Szenarium, das sich auch in den Antichristdramen seit dem Ludus de Antichristo des 12. Jahrhunderts findet. Jede Art von religiöser Devianz wird, nachdem das Christentum integraler Bestandteil der durch Papst und Teufel korrumpierten politischen Ordnung geworden ist, gnadenlos verfolgt und ausgerottet. Der Beginn der Reformation in Wittenberg leitet dann die Heilsund Vollendungsphase der Geschichte ein. Die Vertreter der alten Kirche agieren nun, getrieben von der Bedrohung ihrer weltlichen Macht, als politisch-religiös motivierte Verfolgungsgemeinschaft der >Evangelischen $\triangleleft{ }^{33}$ Die Neuausrichtung der religiösen, sozialen und politischen Ordnung, das von der reformatorischen Bewegung verfolgte Projekt der inneren Christianisierung, der konfessionellen Abgrenzung und der politisch-kulturellen Identitätsfindung ist nicht mehr Thema des Bühnengeschehens. Endzeitgewissheit und Weltgerichtserwartung bilden den Schlussakkord des Stücks, das mit dieser Perspektivierung (fast) abrupt endet. ${ }^{34}$

Thomas Naogeorg spart die Zeit der Reformation aus. Dem Zuschauer ist aufgegeben, das religiös begründete Erstreben einer radikal neuen Ordnung aus seiner aktuellen Situation heraus zu bewerten. Das Publikum ist herausgefordert, das Problem der Kontinuitäten im Negativen, der beabsichtigten Korrektur von Fehlentwicklungen, aber auch den Grad der Umsetzung von religiös-politischen Zukunftsentwürfen in der Phase der Ausdifferenzierung von Konfessionsräumen zu reflektieren. Naogeorg fasst die Reformation als einen radikalen Bruch mit der bisherigen Theologie, den religiösen Praktiken sowie mit dem gesamten Kirchenwesen auf. Die polemische Darstellung der Papstkirche im Pammachius-Drama legt daher einen grundlegenden Umbau von kirchlichen und politischen Strukturen in

33 Deren Sorge um den Machterhalt setzt Naogeorg eindrucksvoll in Szene: So fragt Porphyrius beispielsweise, welche Lehren denn die Evangelischen vertreten würden: >Die Rechtfertigung allein durch den Glauben`, antwortet Dromo. `Weh unseren Bäuchen! , ruft Pammachius daraufhin aus (Naogeorg: Pammachius (s. Anm. 2), lat. vv. 3220-3223; dt. vv. 4261-4264). Oder: Auf die Einzelbeichte und den Tadel von Gewissenslasten sei zu verzichten (Dromo). ,Weh unseren Geldbeuteln! , schreit Pammachius auf (Ebd., lat. 3239; dt. v. 4290).

34 Ne iam expectetis, spectatores optimi, / Ut quintus huic addatur actus fabulae, / Suo quem Christus olim est acturus die (Ebd., vv. 3372-3374); Günstige Herrn euch all ich warnen wil/ Ir wolt nicht warten biß zu disem Spil / Der Fünffte handel wie sich sunst gebưrt/ Werd angehaben und zum end gefurt. (Ebd., vv. 4477-4480). 
den protestantischen Territorien nahe. Die von dem Drama implizit eingeforderte Korrektur und Umsteuerung geht in drei Richtungen: eine Neuordnung des Verhältnisses von kirchlichen Instanzen und weltlicher Herrschaft durchsetzen, einer Neuauflage der Verfolgung von religiöser Devianz enge Grenzen setzen, der Neuausrichtung des religiösen und politischen Lebens an den zentralen Prinzipien der lutherischen Lehre Priorität einräumen. Von der Polemik der frühen Reformationsstücke hebt sich diese Ausrichtung insofern ab, als die in Szene gesetzten Bedrohungsszenarien soziale, politische und religiöse Erfahrungen aus der frühen Phase der Konfessionalisierung voraussetzen. In der Entstehungszeit des Dramas war bereits relativ deutlich, in welcher Weise der neue Glaube auf gesellschaftliche und politische Felder einzuwirken vermochte.

Wie war es um die Realisierung solcher Leitlinien politischen Handelns in der Entstehungszeit des Dramas bestellt? Die grundsätzliche Veränderung der politisch-religiösen Ordnungsparameter ist in den evangelischen Sozialgefügen auch ohne Papst, Kardinäle und Priesterstand in den späten 30er und 40er Jahren des 16. Jahrhunderts als Illusion erkennbar. ${ }^{35}$ Dass der Kultur des Politischen im Prozess der konfessionellen Staatsbildung ein starker religiöser Aspekt verliehen wurde, steht außer Frage. Wenn man davon ausgeht, dass Devianz in einer normativen Ordnung »unterdrückt und verfolgt, d. h. latent gehalten werden ${ }^{36}$ muss, dann überrascht es nur wenig, dass die evangelische Bewegung im Prozess der Konfessionalisierung »die Grundvorstellung von einer öffentlichen, obrigkeitlich geförderten christlichen Verfolgungsgemeinschaft gegenüber religiösen Abweichlern innerhalb der christianitas und gegenüber Juden ${ }^{37}$ beibehalten hat. Naogeorg selbst sah sich mehrfach des Calvinismus verdächtigt und wurde angeklagt, Irrlehren zu verbreiten. ${ }^{38}$

35 Insgesamt war die Reformation- aus heutiger Perspektive - »ein revolutionärer Umbruch [...], der das tradierte obrigkeitliche Machtverständnis eher stabilisierte als schwächte.« Berndt Hamm: Abschied vom Epochendenken in der Reformationsforschung. Ein Plädoyer. In: Zeitschrift für historische Forschung 39 (2012), S. 373-411, hier S. 391.

36 Bernhard Giesen: Latenz und Ordnung. Eine konstruktivistische Skizze. In: Rudolf Schlögl, Bernhard Giesen, Jürgen Osterhammel (Hg.): Die Wirklichkeit der Symbole. Grundlagen der Kommunikation in historischen und gegenwärtigen Gesellschaften. Konstanz 2004, S. 73-100, hier S. 96.

37 Hamm: Abschied vom Epochendenken (s. Anm. 35), S. 389.

38 Dazu Arnold E. Berger: Die Schaubühne im Dienste der Reformation. Erster Teil. Leipzig 1935 (Nachdruck: Darmstadt 1967, S. 221-265 (Thomas Naogeorgus und sein Drama >Pammachius [1538]), hier S. 221-223. Zum `Calvinismus-Vorwurf^vgl. Annemarie Hagmayer: Calvinismus als Etikett. Zuschreibungspraktiken in Leichenpredigten auf sächsische landesherrliche Beamte und Kurfürst Christian I. von Sachsen (1589-1613). In: Eric Piltz, Gerd Schwerhoff (Hg.): Gottlosigkeit und Eigensinn. Religiöse Devianz im konfessionellen Zeitalter. Berlin 2015, S. 149-186. 


\section{Typen bedrohter und restituierter Ordnung}

Abschließend sollen knappe Antworten auf die Frage formuliert werden, welche Formen von bedrohter und von rekonstituierter Ordnung in den Schauspielen imaginiert werden. Wir versuchen ein die Gegensätze zuspitzendes Resümee, das die Durchtränkung religiös-theatraler Deutungsmuster mit ökonomisch-sozialen und politisch-machtbezogenen Gegebenheiten aufnimmt.

1. Die Bedrohung erwächst im Folzschen Fastnachtspiel aus dem vermeintlichen Machtanspruch einer Personengruppe anderen Glaubens in der eigenen kleinräumig-städtischen Gemeinschaft. Die Aufdeckung der konspirativen Absichten wird mit entehrenden Strafen und definitiver Ausschließung der gesamten Gruppe aus dem Sozialverbund geahndet. Eine durch Exklusion ihres negativen Potentials gereinigte (Stadt-)Gesellschaft, so die Perspektive des Stücks, vermag die sozialen und ökonomischen Veränderungen besser zu bewältigen. Ordnung, so könnte man folgern, soll sich stärker an Homogenitätsprinzipien, z. B. an der Vorstellung religiöser Reinheit, orientieren, um den unübersehbaren ökonomisch-sozialen Wandlungsprozessen gewachsen zu sein. Das Szenarium einer Verschwörung der Juden gegen die Christen kann man als Beispiel dafür betrachten, dass ein sozial-ökonomischer Strukturwandel im Schauspiel durch ein Intrigenszenarium verarbeitet wird. Die Komplexität des ökonomischen Zusammenhangs erzeugt offenbar einen diffus mit Finanzgeschäften verknüpften Bedrohungstyp, den man im Horizont städtischer Selbstdeutung ohne weiteres mit dem latenten Bedrohungsszenarium Jüdische Verschwörung verbinden kann.

2. Die exzessive Orientierung von Klerus und Papst an Machterhalt und Eigenwohl bedroht die soziale und ökonomische Existenz zahlreicher Gläubiger, vernachlässigt das Gemeinwohl im eigenen Sozialraum (Stadt) und gefährdet die religiöse Identität der Christenheit insgesamt (Türkengefahr). Das Fastnachtspiel Niklaus Manuels lenkt zudem den Blick auf die Verschränkung zweier Bedrohungsszenarien, auf das Anwachsen der Bedrohung im Außenbereich durch falsche Entscheidungen im Inneren. Diese Bedrohungen können, so die Perspektive des Stücks, durch Einführung des reformatorischen Bekenntnisses in der Stadt und die Entmachtung der kirchlichen Funktionsträger als Voraussetzung für christlich authentisches und politisch kluges Handeln der Führungsschicht abgewendet werden. Die Anhänger des alten Glaubens sind politisch zu entmachten, nicht jedoch aus der sozialen Gemeinschaft radikal auszuschließen oder gar zu vernichten. Es soll eine Ordnung geschaffen werden, in der eine am Gemeinwohl orientierte Religion und Stadtpolitik sich auf ideale Weise zur sstädtischen Kommune als Heilsraum verdichten. Einer im reformatorischen Sinne gereinigten politischen 
Führungsgruppe ist es aufgegeben, die neue Ordnung ohne Aufruhr im Inneren und ohne spaltende Konfrontation in der Eidgenossenschaft durchzusetzen. Trotz der scharfen Ausgrenzung der >Totenfresser«, also des Klerus, zielt das Stück Manuels auf eine Rekonstituierung von Ordnung, die religiöse Devianz weitgehend integriert, solange sie politisch indifferent bleibt. Eine transitorische Situation erschüttert den alten Glauben, ohne gleich eine neue Orthodoxie zu etablieren.

3. Die Macht- und Häresiepolitik der alten Kirche, d. h. die auf Auslöschung zielenden Formen des Umgangs mit religiöser Devianz und Andersheit, stehen im Drama Naogeorgs im Kernbereich bedrohter Ordnung. Die politisch-militärische Entmachtung der alten Kirche und die Akzeptanz der reformatorischen Lehre als heilsrelevante Neuorientierung sind die zentralen Elemente der Bewältigung dieser Bedrohung. Nach ihrer völligen Korrumpierung ist eine grundsätzliche Neukonstituierung der religiös-politischen Ordnung der Welt notwendig. Wie diese Ordnung im Einzelnen aussehen soll, lässt das Pammachius-Drama offen; dass sie jedoch auf den Prinzipien reformatorischen Denkens aufruht, steht außer Zweifel. Erkennbar ist weiterhin, dass der unmittelbare Bezug auf biblische Grundlagen ebenso die Ablehnung von überkommenen kirchlichen Strukturen und Traditionen legitimiert wie die Distanzierung der damit verbundenen politischen und rechtlichen Systeme. Die neue Ordnung sieht von der Geschichte radikal ab, um sich auf Transzendentes zu berufen. Sie wird ebenso als radikaler Bruch wie als Rückkehr zum wiederentdecktenUrsprünglichenimaginiert. NichteinestädtischeKommune ist als Geltungsraum einer solchen Ordnung gedacht, sondern größere territorial-politische protestantisch-geprägte Räume. Jede explizite Charakterisierung der reformierten Ordnung spart das Stück aus, um den Zuschauer dazu zu bringen, die angestrebte neue Ordnung durch den Vergleich mit der dargestellten teuflisch-päpstlichen Ordnung kontrastiv selbst zu durchdenken. 